Review

\title{
Pathophysiology of atrial fibrillation: From initiation to maintenance
}

\author{
Masatoshi Yamazaki, MD, PhD ${ }^{\mathrm{a}, *}$, José Jalife, MD, FHRS \\ a Department of Cardiovascular Research, Research Institute of Environmental Medicine, Nagoya University, Furo-cho, Chikusa-ku, Nagoya 4648601, Japan \\ ${ }^{\mathrm{b}}$ Center for Arrhythmia Research, University of Michigan, Ann Arbor, USA
}

\section{A R T I C L E I N F O}

\section{Article history:}

Received 9 April 2012

Received in revised form 30 April 2012

Accepted 2 May 2012

Available online 30 May 2012

\section{Keywords:}

Atrial fibrillation

Atrial stretch

Persistent atrial fibrillation

Heart failure

\begin{abstract}
A B S T R A C T
Atrial fibrillation (AF) is the most common arrhythmia in adults; it affects approximately 0.8 million patients in Japan alone. Yet despite many years of basic and clinical research, the exact mechanisms underlying the initiation and maintenance of AF remain poorly understood. In this review article, we summarize recent high-resolution optical mapping studies in isolated sheep hearts, which have provided new insights into the dynamics and mechanisms of AF. We focus on 3 models of AF. First, we discuss results from experiments on AF induced by atrial stretch that revealed the presence of spatio-temporally organized waves emerging from the posterior wall of the left atrium. In the presence of adreno-cholinergic stimulation and stretch, AF was governed by evolving interactions between reentry and spontaneous focal discharges. Next, we outline the results obtained from a persistent AF model (average AF duration: 21.3 day) induced by intermittent rapid atrial pacing. By using simultaneous optical mapping of epicardial and endocardial activation patterns, we demonstrated that AF in this model was maintained by 3-dimensional scroll waves with I-shaped filaments anchored to junctions between thin and thick myocardium. Numerical simulation results predicted that wall thickness-dependent activation of stretch-activated channels and the filament tension dynamics were sufficient to explain the specific localization of the I-shaped filament. In a final set of studies discussed herein, we investigated AF in sheep with tachypacing-induced heart failure and found that microreentry in the left atria was a major mechanism of AF maintenance, although focal discharges at the pulmonary vein area also played a role. Large fibrotic patches in failing hearts may serve as potential anchoring sites for micro-reentry in this model. Thus, the 3 different experimental results in isolated sheep hearts presented here clearly suggest that self-sustained rotors do exist in the atria and that such rotors are in fact the high frequency sources that determine the complex patterns of activation that characterize AF.
\end{abstract}

(c) 2012 Japanese Heart Rhythm Society. Published by Elsevier B.V. All rights reserved.

\section{Contents}

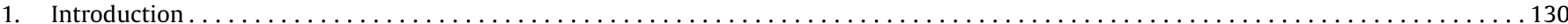

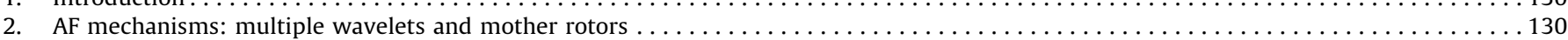

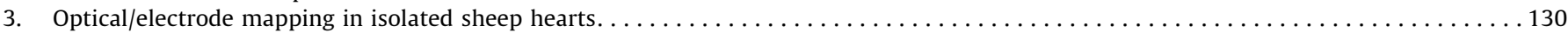

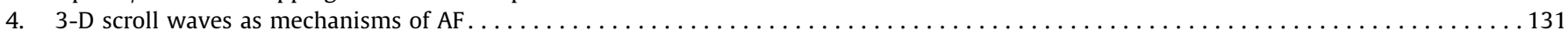

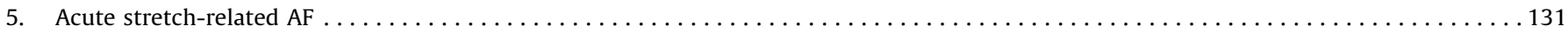

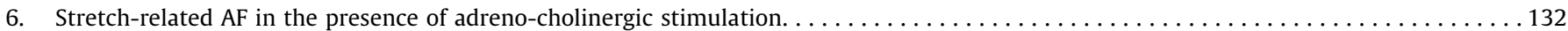

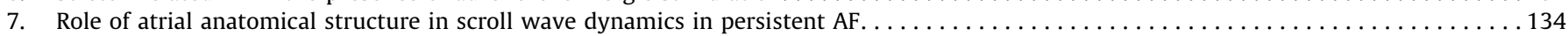

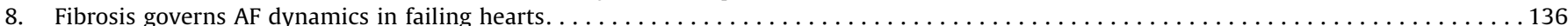

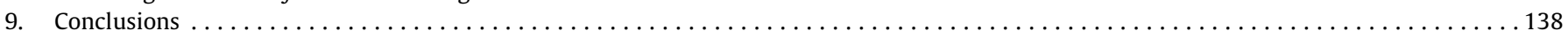

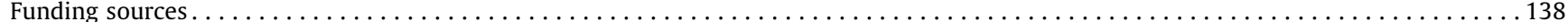

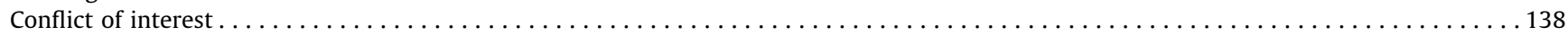

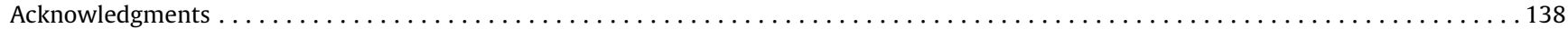

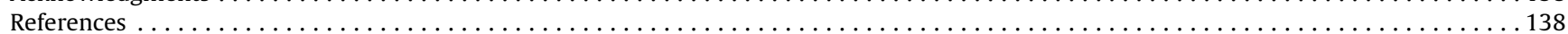

\footnotetext{
* Corresponding author. Tel.: +8152 789 5006; fax: +81527895003.

E-mail address: yamazaki@riem.nagoya-u.ac.jp (M. Yamazaki).
} 


\section{Introduction}

Atrial fibrillation (AF) is the most common arrhythmia in adults and the most common cause of embolic stroke. AF affects approximately 0.8 million patients in Japan alone, and its incidence is expected to grow exponentially [1-3]. However, the electrophysiological mechanisms of the initiation and maintenance of AF remain poorly understood. Pathophysiological changes in atrial structure and function occur as AF progressively advances from an acute to a chronic stage. These alterations include electrophysiological remodeling and fibrosis, [4-6] which give rise to the diverse mechanism of AF pathogenesis. In 1998, Haïssaguerre et al. $[7,8]$ reported for the first time that paroxysmal AF is triggered by focal discharges localized at the pulmonary vein (PV) and that catheter-based procedures that ablate focal triggers effectively terminate up to $90 \%$ of cases of paroxysmal AF. Subsequently, many research investigations confirmed Haïssaguerre's results. As a consequence, PV isolation is widely accepted as a standard therapy for patients with paroxysmal AF, particularly for those without underlying structural heart disease. However, the success rate of PV isolation in patients with persistent $\mathrm{AF}$ is limited, and multiple procedures and the continuation of anti-arrhythmic drug therapy are often required for patients with chronic AF. Furthermore, limitations related to the long duration of the ablation procedure and the occurrence of important side effects renders this approach altogether impractical for the vast majority of AF patients. In addition, one of the major limitations of the currently available ablation techniques is the difficulty of visualizing electrical activity with sufficient spatial resolution to accurately determine the location of potential AF sources. In animal experiments, however, the exploration of the mechanisms linking myocardial stretch, remodeling, and fibrosis to AF $[9,10]$ has benefited from advances in high-resolution electrical and/or optical mapping. In this review article, we summarize recent studies in sheep hearts and discuss the dynamics of wave propagation during AF and its mechanistic link to ionic and structural properties of the atria.

\section{AF mechanisms: multiple wavelets and mother rotors}

Despite many years of research, the precise mechanisms underlying AF initiation and maintenance are far from being understood. In the early 1900s, the two most prevalent explanations for such mechanisms were the "circus movement reentry" and the "ectopic focus" hypotheses [11]. Subsequently, in 1962, Moe et al. [12,13] postulated the "multiple wavelet hypothesis" for AF maintenance, in which disorganized activity of AF may be the consequence of random propagation of multiple independent wavelets (more than 20 wavelets) in a heterogeneous medium of dispersed refractoriness. Later, Allessie et al. [14] provided experimental support for Moe's hypothesis in a dog model of AF induced in the presence of acetylcholine. In that study, several apparently independent wavelets propagated simultaneously throughout both atria. However, unlike Moe's prediction that AF required a minimum number of 26 wavelets to be maintained, Allessie provided a critical number of 4 to 6 wavelets. To our knowledge, this discrepancy has never been resolved. Nevertheless, support for the multiple wavelet hypothesis came also in the form of the so-called surgical maze procedure, in which the highly compartmentalized atria were unable to sustain multiple, randomly propagating wavelets [15]. Thus, the multiple wavelet theory has been widely accepted by most clinical electrophysiologists.

In a landmark article published by Haïssaguerre et al. in 1998, [7] credence was given to rapid spontaneous focal activity in the PV area as the main mechanism of paroxysmal AF initiation and/ or maintenance. Since then, PV isolation by radiofrequency ablation has become the gold standard for the treatment of paroxysmal AF. This, however, is no longer true for persistent AF. Electrical isolation of PV foci is frequently insufficient and additional linear ablation and/or ablation targeting complex fractionated atrial electrograms (CFAEs), which frequently result in the destruction of vast portions of the left atrium, are required [16]. Therefore, the simplistic explanation of such differing outcomes is that focal activity plays a predominant role in paroxysmal $\mathrm{AF}$, whereas persistent $\mathrm{AF}$ is maintained by multiple wavelets.

Different ideas, however, emerged in the 1960s and 1970s in the minds of computational biologists interested in the theory of wave propagation in excitable media. Based on chemical [17] and numerical experiments, [18] scientists in the Soviet Union and the USA began to suggest that fibrillation was not random, but spatiotemporally well organized and maintained by the formation of spiral waves spinning around functional organizing centers called "rotors." Subsequently, the use of optical mapping technology in the early 1990s allowed for the demonstration that a small number of rotors (i.e., 1 or 2 ) were capable of maintaining fibrillation in the atria or ventricles; $[19,20]$ this led to the "mother rotor" hypothesis of AF [21]. Over the last 10 years, several clinical studies have focused on testing whether rotors are the underlying mechanism of AF through inference by the use of dominant frequency mapping [22-24]. Very recently, Narayan et al. [25] developed a phase-mapping approach that enabled them to visualize the dynamics of rotors on the atria of patients through the use of a multiple electrode, basket catheter system. Our work over the last 2 years has focused on the demonstration that electrical activity during sheep persistent AF is organized by a few "scroll waves," 3-dimensional (3-D) waves of excitation emitted by rotors that span from the endocardium to the epicardium within the highest dominant frequency domain [26].

\section{Optical/electrode mapping in isolated sheep hearts}

Since the 1990s, extensive high-resolution optical mapping of the electrical activity during AF in the isolated heart has been an important source of information regarding AF dynamics and its mechanisms [27,28]. To investigate the dynamics of $\mathrm{AF}$, our laboratory selected the sheep heart since its size and electrophysiological properties are similar to those of humans $[29,30]$. Briefly, our experimental procedures were as follows. The hearts were excised and immediately attached to a Langendorff-apparatus. Unless otherwise stated, after a bolus injection of 5 to $10 \mathrm{~mL}$ Di-4-ANEPPS ( $10 \mathrm{mg} / \mathrm{mL}$ ), 2 charge-coupled device (CCD) cameras focused on the epicardium of the left atrial appendage (LAA) and the right atrial appendage (RAA) recorded fluorescence changes, each from an area of $\sim 3 \mathrm{~cm}^{2}$ at 500-1000 frames/s to obtain 5-s movies $(80 \times 80$ pixels $)$. In the simultaneous endocardiumepicardium mapping project, a third CCD camera is connected to a cardio-endoscope introduced in the left atrium by the transseptal route and is synchronized with the other 2 cameras $[26,31,32]$. Dominant frequency (DF) maps are obtained for each optical movie after applying a Fast Fourier Transformation of the fluorescence signal recorded at each pixel. To analyze rotor dynamics quantitatively, we also constructed phase movies after a phase analysis according to that originally described by Gray et al. [33] but modified as to be based upon Hilbert transformation as in the report by Warren et al. [34]. Bipolar electrodes were positioned on the left and right atrial appendages, on the left atrial roof, inside the coronary sinus and on the left superior pulmonary vein (LSPV), left inferior pulmonary vein (LIPV), right superior pulmonary vein (RSPV), and right inferior pulmonary 
vein (RIPV). During AF, bipolar electrograms were acquired at a sample rate of 200 points per s. Segments of 4096 points (approximately $20 \mathrm{~s}$ ) were analyzed with Fast Fourier transformation as previously reported, [35] and the highest dominant frequency location (DFmax) was determined.

\section{3-D scroll waves as mechanisms of AF}

The analysis of the general characteristics of reentrant activity by means of optical mapping is usually achieved in 2 dimensions, i.e., spiral wave reentry. However, accurate understanding of the mechanisms of reentry in the whole heart requires knowledge of the manner in which electrical waves propagate in $3-D$, as the myocardial wall thickness is not insignificant. Therefore, ideally, it would be important to elucidate the dynamics of votex-like reentrant activity, i.e., scroll waves spanning the thickness of the atrial wall. While new approaches such as laminar optical tomography are enabling investigators to visualize more deeply into the myocardial wall, [36] most recordings of fibrillatory wave propagation to date have been performed from the epicardium or the endocardium. Hence, the experimental knowledge regarding scroll waves in the heart remains very limited, despite significant progress in the theory and computer modeling of 3-D votex-like reentrant activity [37,38].

Examples of numerically generated vortices and their organizing centers in 3-D excitable media are illustrated in Fig. 1. Panels a to d show 4 different configurations of 3-D scroll waves that were computed using the FitzHugh-Nagumo model [39,40]. Panel a shows an example of the simplest configuration of a scroll wave, which rotates around a central I-shaped filament spanning the entire myocardial wall. As shown in panel $b$, the filament of the scroll waves can bend into an L-shape that appears as a single spiral wave on 1 surface of the excitable medium, and breakthrough or 1-way propagation on the opposite surface. Panel c shows an example of a U-shaped filament scroll wave that is identified when a "figure-of- 8 " is seen on the surface and likely a breakthrough or 1-way propagation on the opposite surface. The variety of 3-D configurations may set a stage for diverse dynamic behaviors of scroll waves, even in homogeneous myocardial tissue. Under conditions of normal excitability, the filament of scroll waves is subjected to the action of "positive tension" and tends to shrink. On the other hand, under low excitability conditions, the filament is subjected to "negative tension" and tends to enlarge. Such phenomena have been predicted in a modeling study, [38] and we first confirmed them experimentally in isolated sheep hearts by using a simultaneous endocardial and epicardial high-resolution optical mapping setup [26]. In these experiments, 3 CCD cameras and a cardio-endoscope enabled simultaneous recording from the epicardium of the RAA, and the LAA epicardium and endocardium.

Fig. 2 illustrates an example of an atrial scroll wave with an I-shaped filament, which rotated clockwise when viewed from the epicardial surface and counterclockwise when viewed from the endocardial surface. Phase maps of the 2 recording surfaces made it possible to detect concurrent epicardial and endocardial phase singularities (PSs) around which functional reentry was organized. Interestingly, I-shaped filament scroll waves are generally stable and long lasting, with minimal twisting under normal conditions since their filaments are subject to positive tension.

\section{Acute stretch-related AF}

AF is commonly associated with acute atrial dilatation and stretch $[9,10,41,42]$. In patients with acute myocardial infarction, heart failure and/or mitral valve disease, atrial dilatation and stretch have been widely recognized as the major pathophysiological factor of $\mathrm{AF}$ initiation and maintenance. Despite many studies demonstrating a significant relationship between stretch of the posterior left atrium (PLA) and PV and the dynamics of AF, $[43,44]$ a clear role of stretch-activated channels (SACs) in these regions has not yet been established. Activation of SACs that have an ohmic IV relationship (i.e., linear voltage dependency of the ion channel conductance) causes a shortening of the action potential duration (APD) at $50 \%$ repolarization $\left(A P D_{50}\right)$, a prolongation of $\mathrm{APD}$ at $90 \%$ repolarization $\left(\mathrm{APD}_{90}\right)$, and a depolarization of the
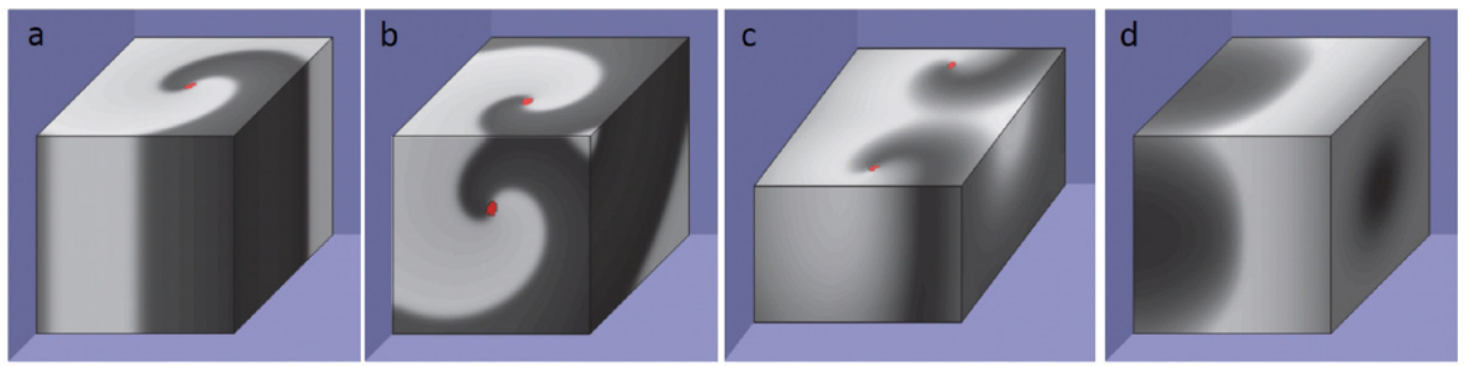

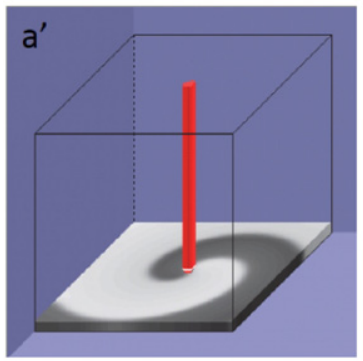

I-shaped

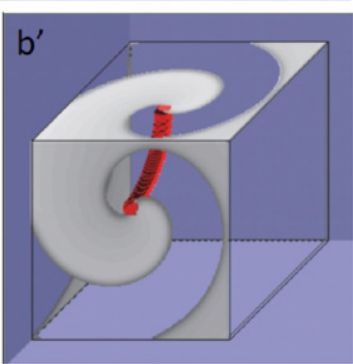

L-shaped

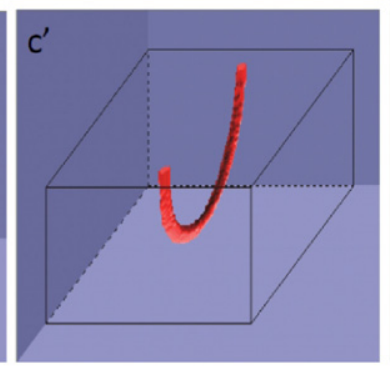

U-shaped

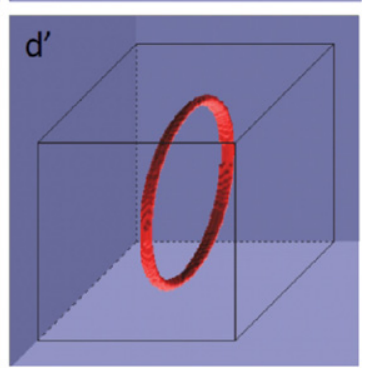

O-shaped

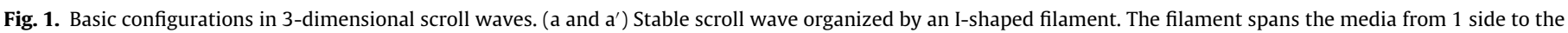

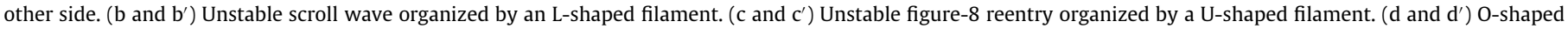
filament scroll wave. Red lines indicate the filaments of a 3-dimensional scroll wave.

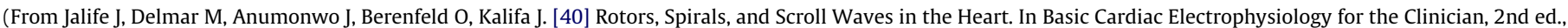
Wiley-Blackwell, Oxford, UK; 2009. p. 191-222, with permission). 


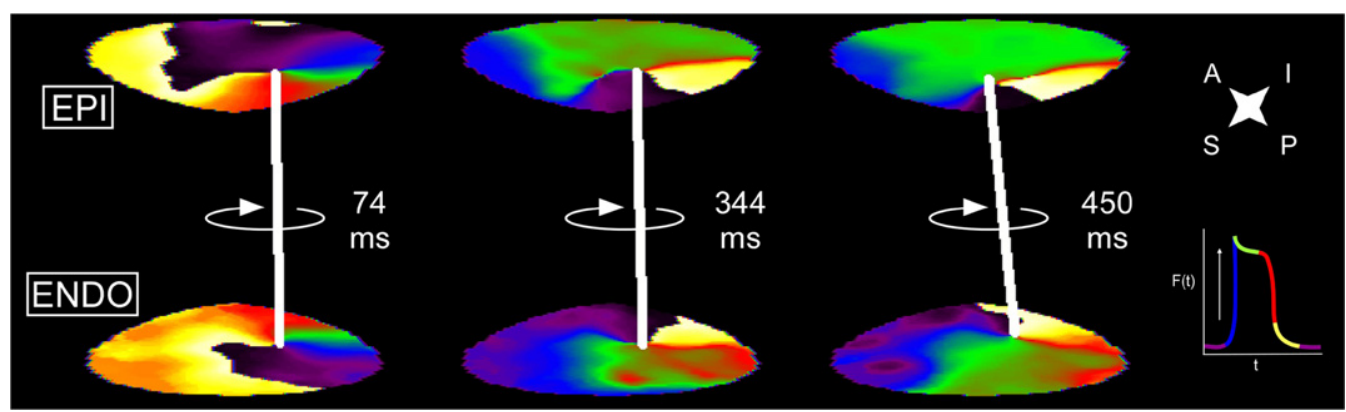

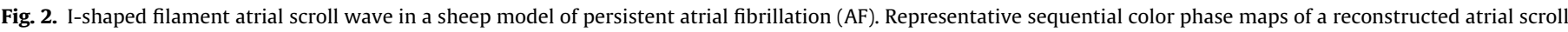

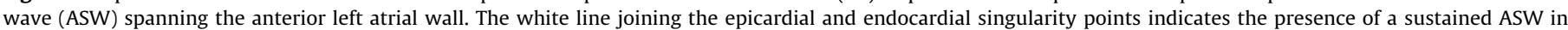
which the I-shaped filament rotates clockwise during $450 \mathrm{~ms}$.

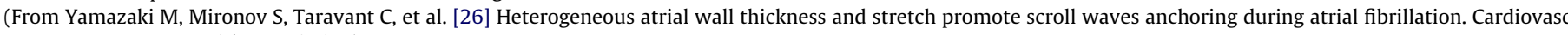
Res. 2012;94:48-57, with permission).

resting membrane potential, which may potentially result in spontaneous activity. To investigate the hypothesis that atrial dilatation is associated with arrhythmogenic sources at the PVs, we implemented in a well-characterized acute stretch-related AF (SRAF) model in sheep $[45,46]$ that was originally described by Ravelli et al. [47] in a rabbit model. In this model, the intra-atrial septum was perforated and all venous orifices were closed with the exception of the inferior vena cava, which was connected to a cannula controlling the level of intra-atrial pressure (IAP). As reported previously, [45,46] sustained episodes of AF ( $>1 \mathrm{~h}$ ) were reproducibly induced by burst pacing when the IAP was raised to more than $10 \mathrm{~cm} \mathrm{H}_{2} \mathrm{O}$.

In Fig. 3, panels $A$ and $B$ depict representative DF maps from the LA free wall (LAFW) and the PLA, including the junction to the left superior PV (LSPV) during SRAF induced at IAPs of 18 and $5 \mathrm{~cm} \mathrm{H}_{2} \mathrm{O}$, respectively. At $18 \mathrm{~cm} \mathrm{H}_{2} \mathrm{O}$, the DF of fibrillatory activity during SRAF was higher in the PLA than in the LAFW (panel A). In contrast, the DF was roughly similar in both regions at an IAP of $5 \mathrm{~cm} \mathrm{H}_{2} \mathrm{O}$ (i.e., atrial flutter). Panel $\mathrm{C}$ shows representative signals recorded from the PLA (site a) and LAFW (site b) during SRAF at an IAP of $18 \mathrm{~cm} \mathrm{H}_{2} \mathrm{O}$. The cycle length of $\mathrm{AF}$ activity was shorter in the PLA (site a) than in the LAFW (site b). Panel $\mathrm{D}$ summarizes $\mathrm{DF}_{\max }$ data from 8 experiments: At IAP $>10 \mathrm{~cm} \mathrm{H} \mathrm{H}_{2} \mathrm{O}$, the PLA $\mathrm{DF}_{\max }(12.0 \pm 0.2 \mathrm{~Hz})$ was significantly higher than the LAFW $\mathrm{DF}_{\max }(10.5 \pm 0.2 \mathrm{~Hz}, p<0.001)$, whereas at IAP less than $10 \mathrm{~cm} \mathrm{H}_{2} \mathrm{O}$ there was no significant difference in the PLA DF max $(10.8 \pm 0.3 \mathrm{~Hz})$ and LAFW DF $\max (10.2 \pm 0.3 \mathrm{~Hz})$. Notably, these DF $_{\max }$ values in the PLA and LAFW were appreciably higher than in the RAA $(7.8 \pm 0.3 \mathrm{~Hz})$. These results strongly suggest that a high frequency AF driver exists in the PLA near the junction to the PVs in SRAF. An analysis of spatio-temporal excitation patterns during SRAF revealed that the normalized number of spatio-temporally periodic waves in the PLA was strongly correlated with $\operatorname{IAP}(p=0.002)$. Furthermore, a significant positive correlation was found between IAP and the number of waves appearing from the LSPV junction in the PLA $(p=0.02)$, but not with those emanating from the LAFW $(p=0.09)$.

The electrophysiological mechanisms underlying high frequency activity observed in the PLA during SRAF could be reentry (rotor) or focal discharges (triggered activity). To discriminate between these mechanisms, we perfused ryanodine $(10-40 \mu \mathrm{M})$ or caffeine $(5 \mathrm{mM})$ to abolish calcium overload-mediated triggered activity during SRAF [46]. These pharmacological interventions successfully terminated a majority of SRAF (in 10/13 animals), whereas SRAF persisted despite drug application in the remaining cases (in 3/13 animals). These results suggest that SRAF in normal sheep hearts may be predominantly maintained by spontaneous focal discharges, although it should be noted that reentrant activity could also play a significant role in some cases.
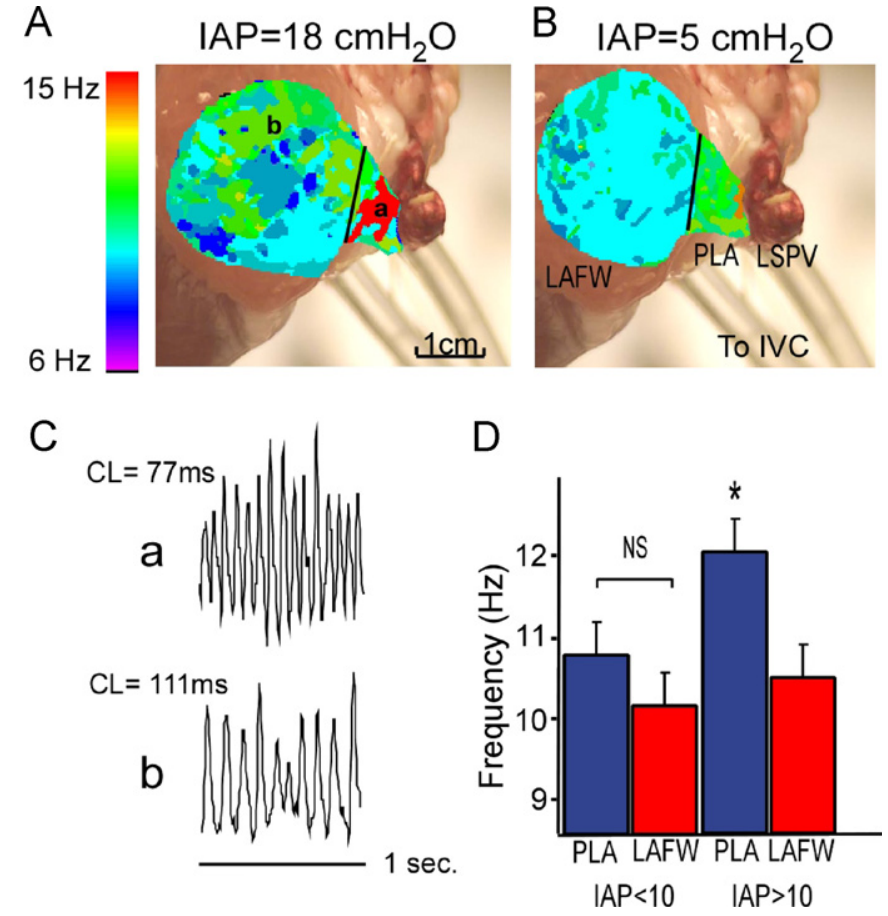

Fig. 3. Effects of acute atrial stretch on atrial fibrillation dynamics at the posterior left atrium. (A and B) Dominant frequency (DF) maps from a single heart at intraatrial pressures (IAP) of 18 and $5 \mathrm{~cm} \mathrm{H}_{2} \mathrm{O}$, respectively. DF maps are superimposed on a color picture of a heart for illustrative purposes. (C) Single-pixel recordings from the junction of the pulmonary vein (JPV) and the left atrial free wall (LAFW) at $30 \mathrm{~cm} \mathrm{H}_{2} \mathrm{O}$. IVC indicates inferior vena cava; $\mathrm{CL}$, cycle length. (D) Bar graph showing $\mathrm{DF}_{\text {Maximum }}($ mean \pm SEM) in the JPV (blue) and LAFW (red) at IAPs $<10$ and $>10 \mathrm{~cm} \mathrm{H}_{2} \mathrm{O}\left({ }^{*} p<0.001\right)$.

(From Kalifa J, Jalife J, Zaitsev AV, et al. [45] Intra-atrial pressure increases rate and organization of waves emanating from the superior pulmonary veins during atrial fibrillation. Circulation. 2003;108:668-671, with permission).

\section{Stretch-related AF in the presence of adreno-cholinergic stimulation}

Similar to atrial stretch, autonomic input to the heart is important in AF initiation and maintenance, [48-50] but until recently the exact mechanisms whereby AF is maintained in the continuous presence of adreno-cholinergic stimulation remained unexplored. Recently, it has been shown that AF could be terminated by application of radiofrequency current catheter ablation to atrial areas containing cardiac ganglionated plexi, which function as modulators of autonomic input to the heart [51]. In addition to increasing trans-sarcolemmal calcium influx through the L-type calcium channel $\left(I_{\mathrm{Ca}, \mathrm{L}}\right)$, adrenergic stimulation gives rise to intracellular calcium overload. On the other hand, 
cholinergic stimulation of muscarinic receptors opens the acetylcholine-activated potassium channel $\left(I_{\mathrm{K}, \mathrm{ACh}}\right)$, resulting in abbreviation of the atrial action potential duration. Recent studies suggest that simultaneous activation of adrenergic and cholinergic nerves (adrenocholinergic stimulation, ACS) causes delayed afterdepolarizations (DADs) [50] and late phase-3 early afterdepolarizations (EADs) $[48,52]$ through the activation of the forward mode of the sodium/ calcium exchanger, thus resulting in focal discharges being expected to promote $\mathrm{AF}$ initiation and maintenance.

We investigated the consequences of autonomic modification of SRAF in isolated sheep hearts by perfusing acetylcholine $(1 \mu \mathrm{M})$ and isoproterenol $(0.03 \mu \mathrm{M})$ simultaneously. Fig. $4 \mathrm{~A}$ and B show a representative example of the activation pattern in the LAFW (phase map snap shots) during 2 SRAF episodes induced in the setting of ACS. In Fig. 4A, breakthrough resulted in wavebreak and rotor formation. In this case, the breakthrough (appearing at $4159 \mathrm{~ms}$ ) that emerged on the upper LA appendage underwent wavebreak, thus leading to the formation of a counterclockwise rotor with a cycle length of $72 \mathrm{~ms}$ equaling $1 / \mathrm{DF}_{\max }$. On the other hand, while several rotors could be observed during a given $\mathrm{AF}$ episode, most were forced to drift and eventually terminate after 3 to 4 rotations by their interaction with the repetitively

\section{SRAF+ACS}
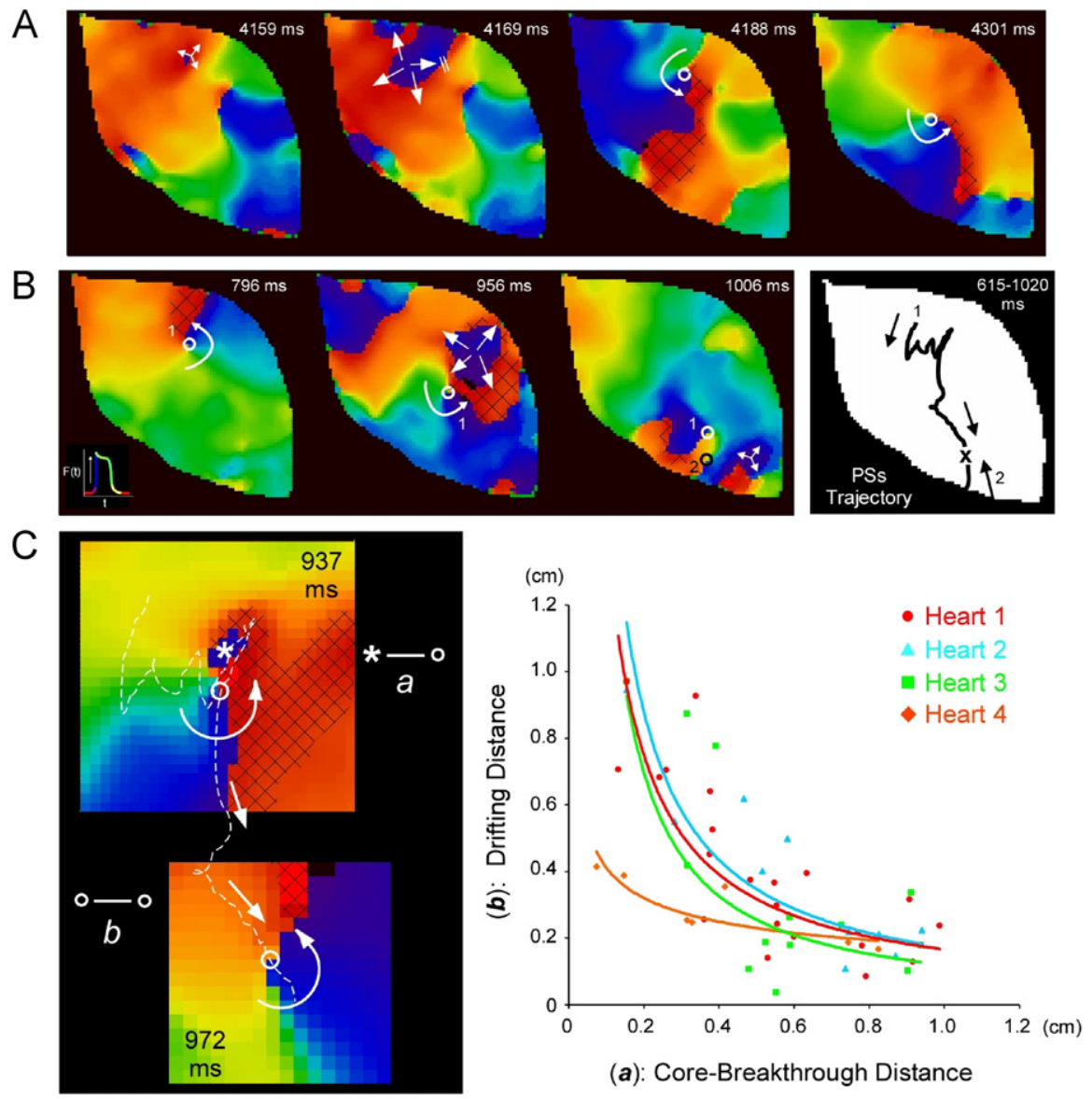

(a): Core-Breakthrough Distance
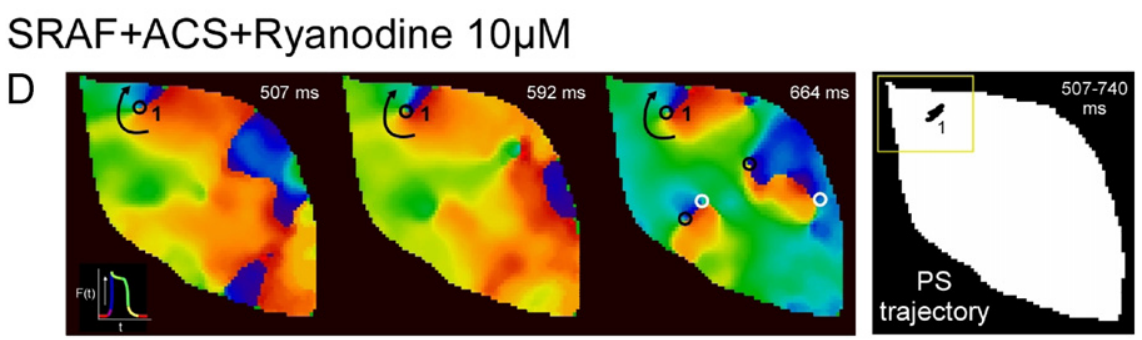

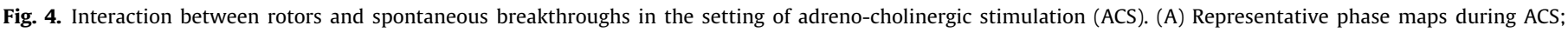

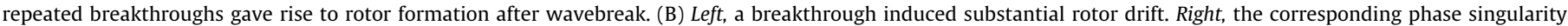

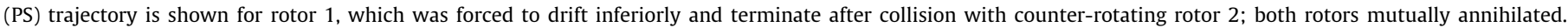

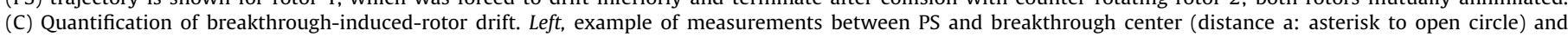

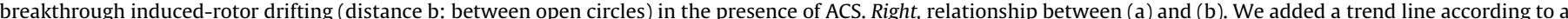

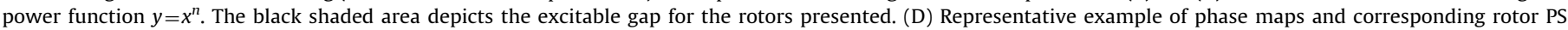

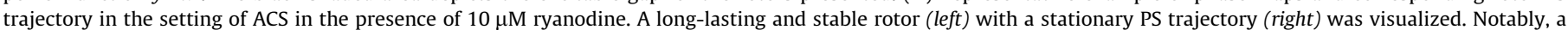

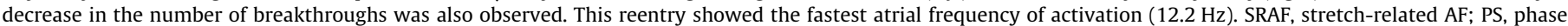
singularity.

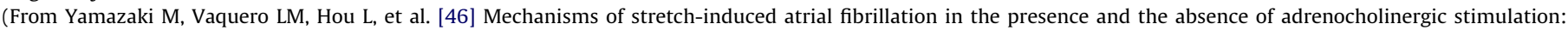
interplay between rotors and focal discharges. Heart Rhythm. 2009;6:1009-1017, with permission). 
emerging breakthroughs. In Fig. 4B, the breakthrough that appeared near the center of the field of view at $956 \mathrm{~ms}$ forced a rotor to drift over a long distance toward the LA roof. Then, collision of this rotor with a second rotor led to its annihilation. These results suggest that SRAF mechanisms in the presence of ACS resulted from a constant interaction between rotors and spontaneous focal discharges.

We measured the substantial drifting distance that rotors underwent during SRAF in the setting of ACS. We traced the trajectory of phase singularities (PSs) at the rotor tip and quantified the spatio-temporal relationship between PSs and the breakthrough site as follows: first, the PS-to-center of breakthrough distance at the onset of the breakthrough was noted as distance (a; i.e., from the asterisk to the white circle in the upper panel in Fig. 4C). Distance (a) was then plotted against the PS-tocenter value of the breakthrough distance after the rotor had undergone a half rotation; the latter was noted as distance (b) and measured between the white circles in the top and bottom panels. As plotted on the right graph $(n=4)$, there was a nonlinear inverse relationship between rotor drifting and PS-breakthrough distance. Breakthroughs caused the most drifting when they occurred in the immediate vicinity of the PS, and the drifting effect tended to fade as the PS-breakthrough distance increased above $0.6 \mathrm{~cm}$. Interestingly, when spontaneous focal discharges were abolished, the rotors were long lasting and no longer drifted.

Fig. 4D illustrates a long-lasting and relatively stable rotor with a stationary PS trajectory after perfusion of ryanodine $(10 \mu \mathrm{M})$, which was used to abolish triggered activity in the setting of ACS. Taken together, these results indicate that in the presence of ACS, SRAF is governed by an evolving interplay between reentry and spontaneous focal discharges.

\section{Role of atrial anatomical structure in scroll wave dynamics in persistent $A F$}

Previous studies [53-55] have suggested that atrial pectinate muscle ridges could be the site of conduction delays, wavebreaks, and reentry initiation. Computer simulations have shown that wavebreak and scroll wave dynamics are affected by anatomical factors such as myocardial thickness and fiber direction [37-39]. Furthermore, in various experimental models and in humans, it was clearly demonstrated that both ionic and structural remodeling lead to AF initiation and maintenance [4,5,56,57]. In addition, recent studies $[58,59]$ have suggested that prolonged atrial tachypacing increases interstitial fibrosis and enhances endocardial-epicardial electrical dissociation, resulting in $\mathrm{AF}$ perpetuation.

We explored the roles of atrial structure in controlling the dynamics of persistent AF (PtAF; average duration: $21.3 \pm 11.9$ day) induced in sheep by prolonged intermittent atrial tachypacing [26] $(20 \mathrm{~Hz}$ in $30-\mathrm{s}$ periods) for $1-7$ weeks. The LA was significantly dilated from $24.7 \pm 0.2 \mathrm{~mm}$ in controls to $30.7 \pm 0.6 \mathrm{~mm}$ in remodeled hearts $(p<0.05)$. Optical imaging was carried out during acute SRAF induced in isolated hearts from PtAF animals. To reveal in detail the relationship between the atrial anatomical structure and the location of rotors and PSs, simultaneous recordings were performed from the LAA endocardium and epicardium, the RAA, and the PLA by using 3 CCD cameras and a steerable cardio-endoscope.

We evaluated and classified the activation patterns at the region showing $\mathrm{DF}_{\max }$ in the $\mathrm{LAA}$. The vast majority of LAA patterns included I-shaped filament scroll waves (31/42 AF waves, $n=5)$ in PtAF hearts, while centrifugal breakthroughs (19/43 AF waves) and spatiotemporal organized wavefronts (13/43 AF waves) were the most frequent in hearts without PtAF $(n=5)$. Fig. $5 \mathrm{~A}$ shows a representative example of a $3-\mathrm{D}$ atrial scroll wave with an I-shaped filament during SRAF in a heart with PtAF. Four consecutive phase movie snapshots were superimposed with the corresponding high-resolution anatomical pictures. These 3-D scroll waves that span the LA wall from epicardium to endocardium are surprisingly stable. Fig. 5B shows the tracing of the I-shaped filament trajectory. White lines on the epicardium and endocardium indicate the trajectory of PSs. From 20-64 ms, the filament remained anchored to thin myocardium bordered by thick pectinate muscles. Between 64 and $140 \mathrm{~ms}$, the
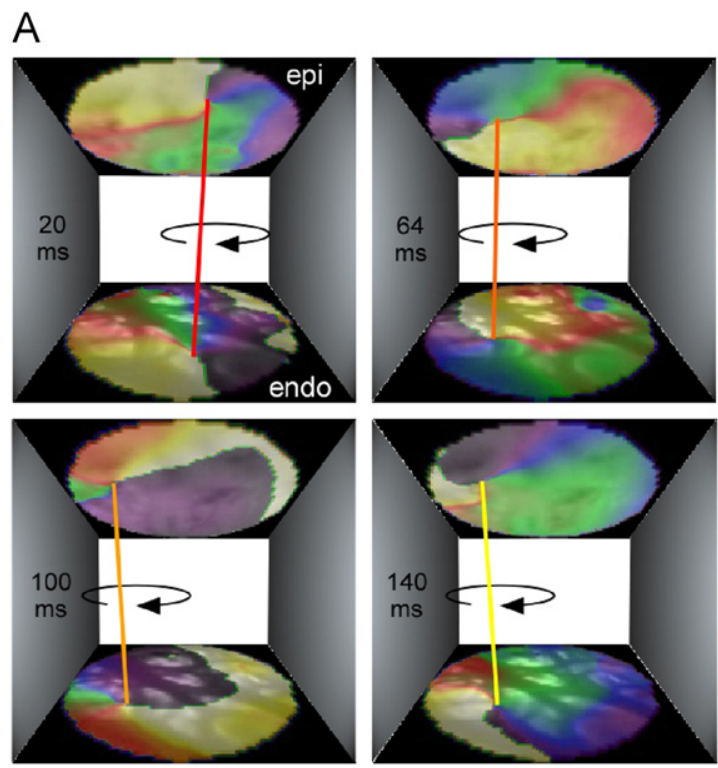

B

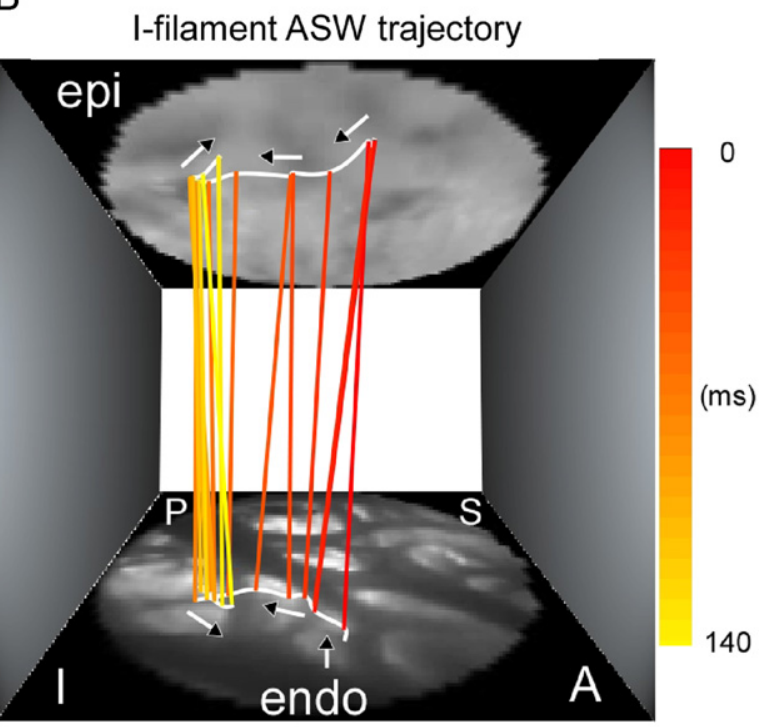

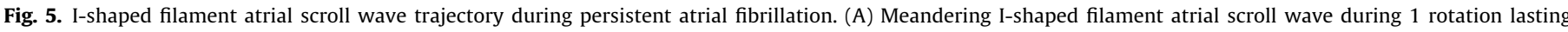

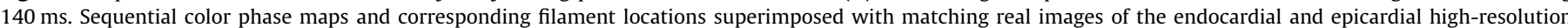

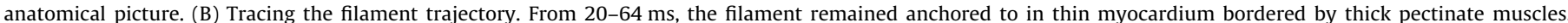
Between 64 and $140 \mathrm{~ms}$, the filament drifted across a pectinate muscle segment before anchoring to a neighboring island of thin myocardium.

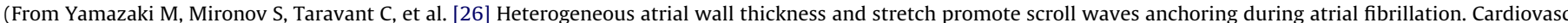
Res. 2012;94:48-57, with permission). 
filament drifted across a pectinate muscle segment before anchoring to a neighboring island of thin myocardium. Taken together, these 3-D scroll waves that maintained SRAF were confined to specific anatomical structures in remodeled hearts with PtAF.

We compared the dynamics of rotors in 4 different settings: AF induced in the presence or absence of acute atrial stretch in normal $(\mathrm{NH})$ or remodeled hearts with PtAF. In this series of experiments, AF was induced at an IAP of $12 \mathrm{~cm} \mathrm{H}_{2} \mathrm{O}$ (stretch, $\mathrm{S}$ ) or $5 \mathrm{~cm} \mathrm{H}_{2} \mathrm{O}$ (non-stretch, NS). Fig. $6 \mathrm{~A}$ demonstrates an example of representative phase maps and the trajectories of PSs in AF episodes with and without stretch in a heart with PtAF. In PtAF with stretch (top), a long-lasting rotor with stationary PS trajectory showing minimal drift was observed, whereas a rotor in PtAF without stretch (bottom) showed prominent drift; it appeared at the right upper corner of the LAA and quickly drifted to far region before disappearing from the field of view. Fig. $6 \mathrm{~B}$ summarizes the rotor dynamics in 4 settings. The drifting distance of the rotor core in PtAF with stretch $(\mathrm{S})$ was significantly lower compared with NH with stretch (S) and PtAF without stretch (NS). Moreover,

A PtAF with stretch $\left(12 \mathrm{cmH}_{2} \mathrm{O}\right)$

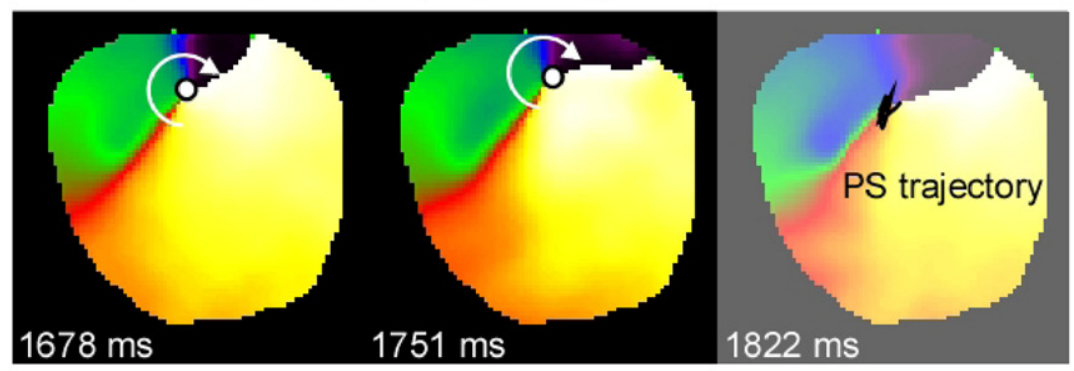

PtAF without stretch $\left(5 \mathrm{cmH}_{2} \mathrm{O}\right)$
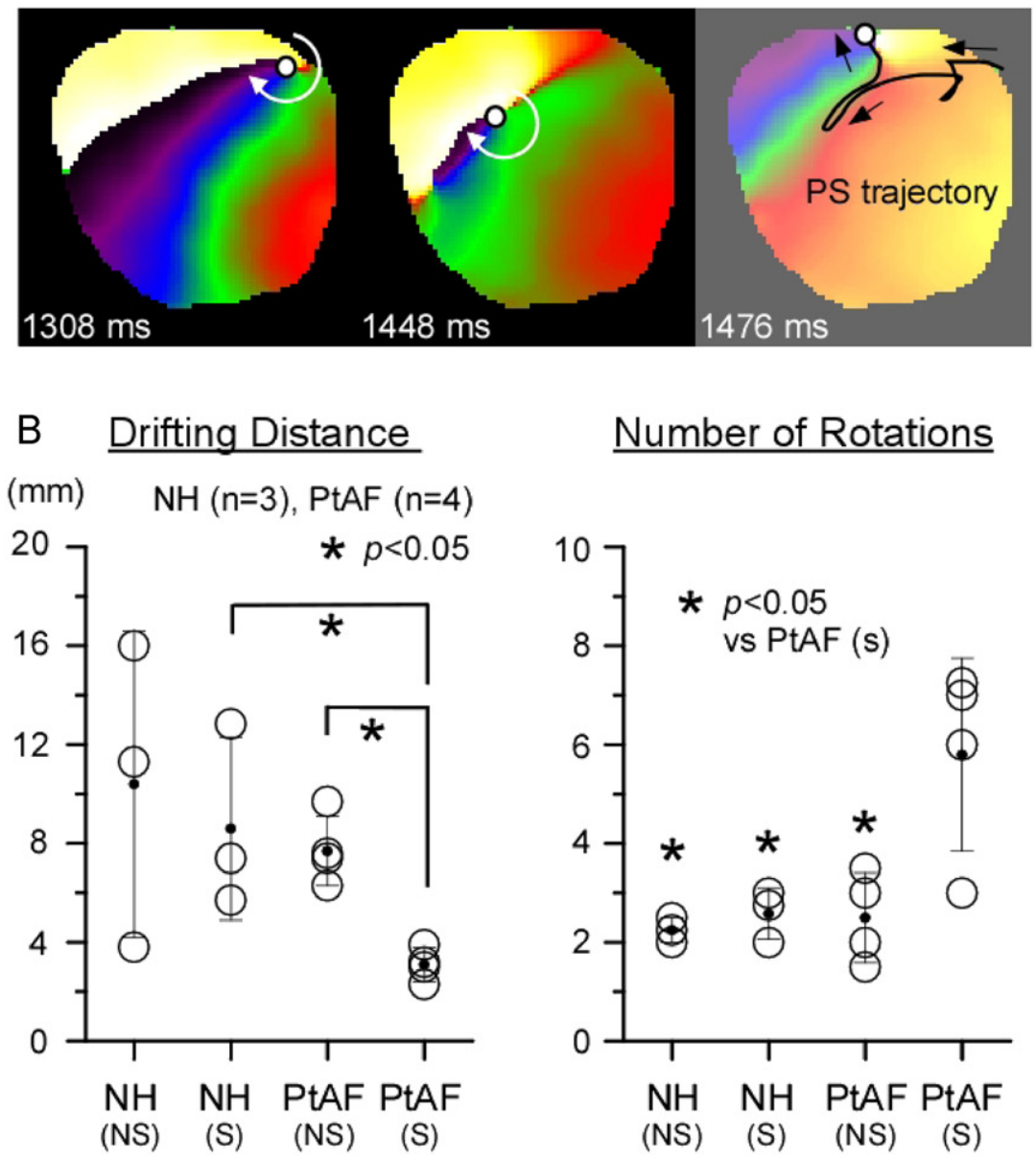

\section{Number of Rotations}

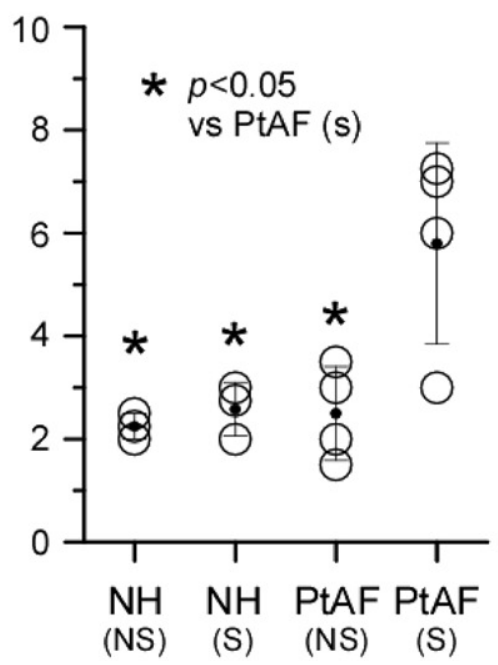

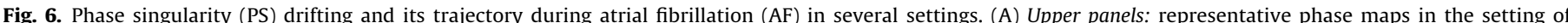

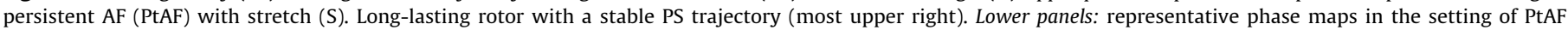

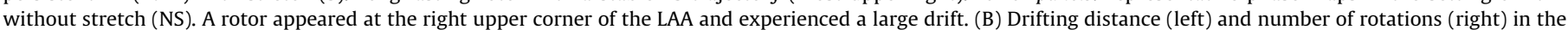

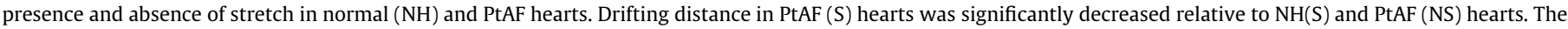
number of rotations was significantly larger in PtAF (S) than in NH (NS and S) and PtAF (NS) hearts.

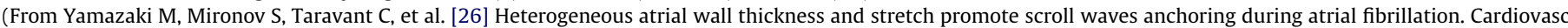
Res. 2012;94:48-57, with permission). 


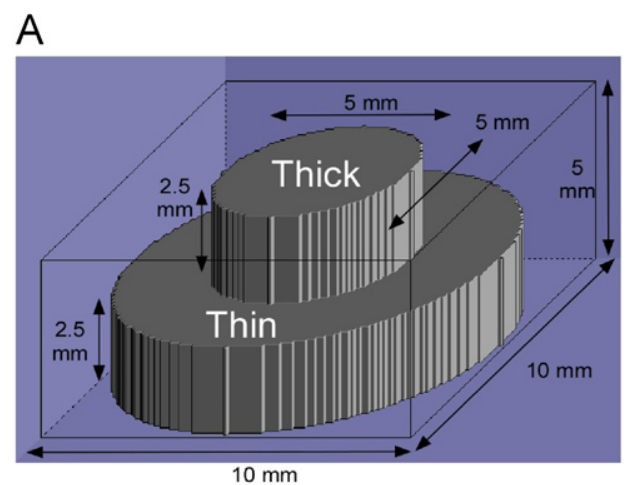

B

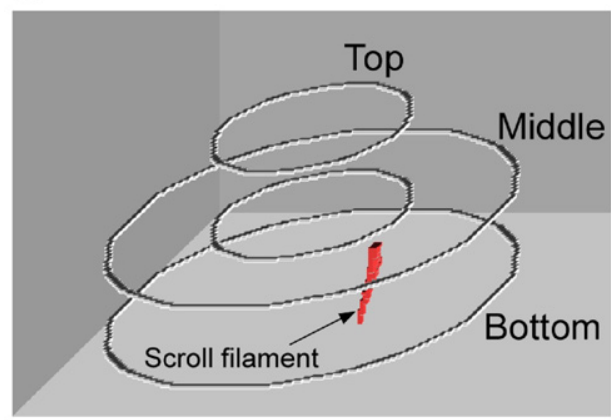

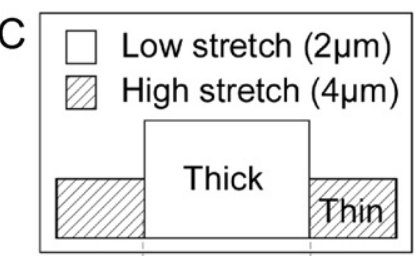
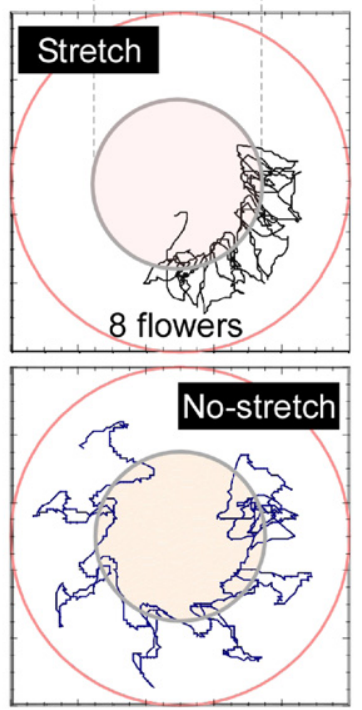

Filament trajectory

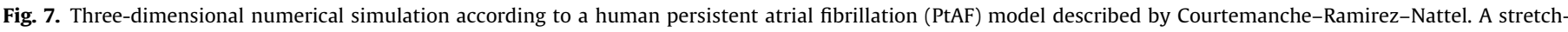

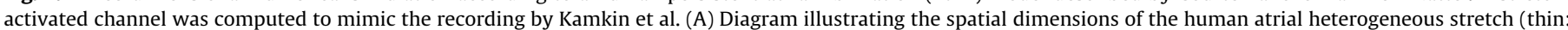

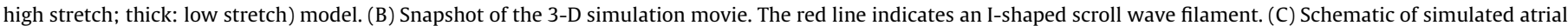

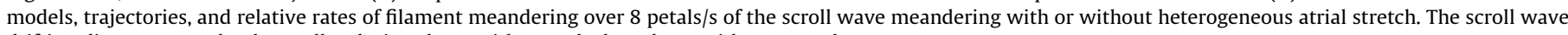
drifting distance was clearly smaller during those with stretch than those without stretch.

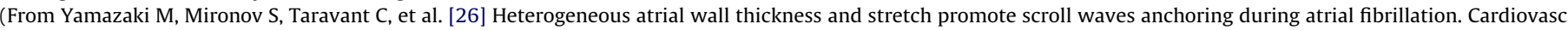
Res. 2012;94:48-57, with permission).

the number of rotations in PtAF with stretch (S) was significantly higher compared with that of the other groups.

The results described thus far suggest that PtAF-induced atrial remodeling combined with acute atrial stretch facilitates anchoring of 3-D scroll waves to the border of atrial pectinate muscle and prolongs their life span in favor of AF maintenance. However, why is it that scroll waves anchor to the edges of thick muscle bundles such as pectinate muscles? To answer this question, we implemented a simplified 3-D numerical simulation of the human PtAF model using the equations of Courtemanche-RamirezNattel et al. [60,61]. Two levels (high, $4 \mu \mathrm{m}$; low, $2 \mu \mathrm{m}$ ) of stretch-activated channels were computed to mimic recordings documented by Kamkin et al. [62]. In this simulation, the effects of stretch-activated channels, such as depolarization of the resting potential, were greater in the thin segments. Fig. 7A presents a diagram showing the spatial dimensions of the atrial model. Fig. 7B shows an example of an I-shaped filament (indicated by a red dotted line) scroll wave. I-shaped filament scroll waves induced by S1-S2 stimulation were mainly located at the junction between thin and thick myocardial segments. One theoretical explanation could be the "minimal length principle" described by Wellner et al. [38] which states that the filament of a scroll wave tends to drift to areas with conditions contributing to minimize its length. For instance, in thick myocardium, the conduction velocity is normal and scroll wave filaments are subject to positive tension, resulting in a strong tendency to drift toward the thinnest myocardial segments. Conversely, in thin myocardium, increased wall tension leads to a preferential activation of stretchactivated channels, leading to depressed conditions of impulse propagation. Consequently, scroll wave filaments in thin regions are exposed to negative tension and tend to drift toward the thickest myocardial segments. Such results suggest that scroll wave filaments are submitted to opposite drifting forces resulting from the heterogeneity of impulse velocities in thin and thick myocardium. Thus, as depicted in Fig. $7 \mathrm{C}$, the balance between strong positive tension in thick regions and negative tension in thin regions could explain why I-filament scroll waves are preferentially located at the junction between thin and thick myocardium.

\section{Fibrosis governs AF dynamics in failing hearts}

It is well known that heart failure increases the incidence of atrial tachyarrhythmias, resulting in a significantly higher rate of morbidity and mortality [63]. Heart failure induced by rapid pacing leads to electrical as well as structural remodeling of atrial muscle, and the latter is characterized by extensive atrial interstitial fibrosis [64]. Atrial interstitial fibrosis associated with heart failure may promote $\mathrm{AF}$ maintenance even in the absence of atrial stretch [65]. In the fibrotic atria of transforming growth factorbeta (TGF- $\beta$ ) transgenic mice and in dogs with pacing-induced heart failure, slower and more organized atrial tachyarrhythmias were documented [66,67]. In our recent preliminary study, [68] we induced heart failure by ventricular tachypacing $(220 \mathrm{bpm})$ in sheep and investigated the dynamics and mechanisms of AF. Echocardiography confirmed the development of congestive heart failure and a significant increase of the LA size (from $31 \pm 1$ in controls to $50 \pm 1 \mathrm{~mm}$ in failing hearts) at $6-8$ weeks after pacing. Then, we conducted optical mapping in the isolated hearts by recording from the endocardial surface of the PLA, which was 
exposed by a minimal incision applied to the LA. The DF of excitation during sustained $\mathrm{AF}$ episodes induced in the presence of 0 to $4 \mu \mathrm{M}$ acetylcholine was much slower in failing hearts than in controls; importantly, in both cases the $\mathrm{DF}_{\max }$ at the PLA was significantly larger than the DFs of bipolar electrograms recorded from the Bachmann's bundle (BB) and RAA (Fig. 8A).

Fig. 9A shows sequentially obtained phase maps from a representative experiment during an AF episode. Fig. 9B shows a schematic of the breakthrough sites (color dots) in the PLA during AF induced without acetylcholine in failing hearts. In all of the hearts that were examined, the majority of breakthrough sites were located at the periphery of the PLA. In failing hearts, the activation patterns were mainly repetitive breakthroughs appearing at the PV ostium and propagating toward the periphery of the field of view (Fig. 9A); in some cases, long-lasting stationary micro-reentrant waves were also observed, which anchored to the PVs (Fig. 9C and D). The biatrial electrograms obtained by recording differentially from both atrial appendages (LAA and RAA) showed slower and irregular activity, whereas the PV electrograms were monomorphic and highly periodic, suggesting that a stable source existed within the PV ostial region. Histological analysis of the PLA revealed that interstitial fibrosis was significantly greater in failing hearts compared with controls (Fig. 8B). More importantly, the fibrotic areas tended to cluster in relatively large patches in failing hearts, but not in controls. In addition, the fibrotic patches observed in failing hearts were preferentially located in the periphery of the PLA close to the PV ostium. Thus, large patches of fibrosis may have the ability to anchor micro-reentrant sources in failing hearts. Taken together, these preliminary data suggest that the

A

B
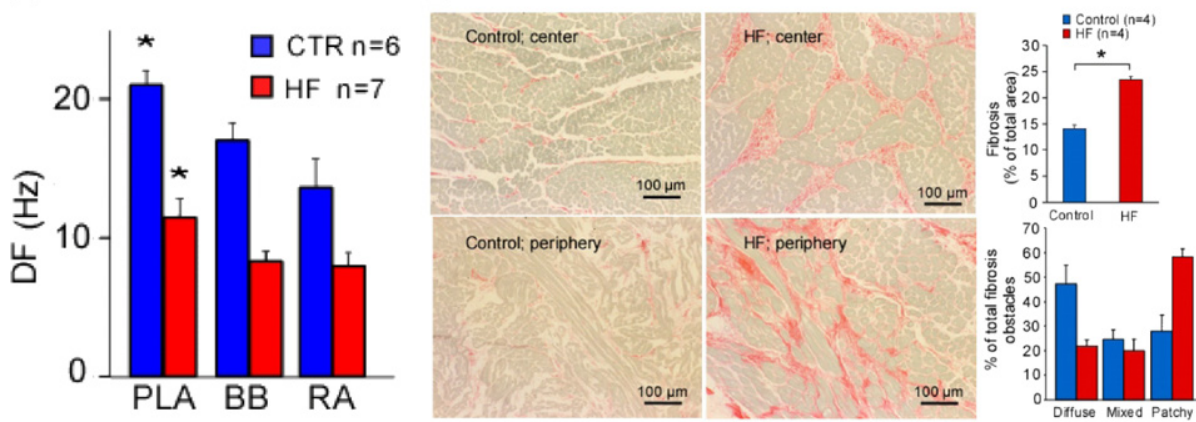

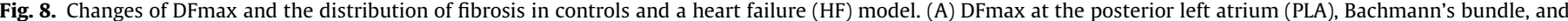

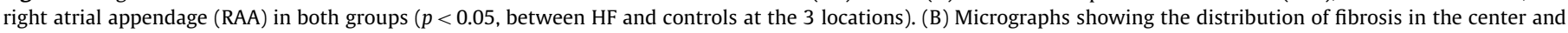

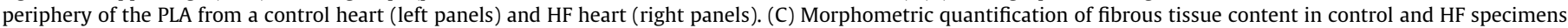
$(p<0.001)$.

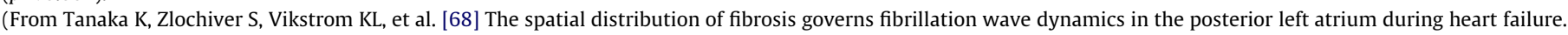
Circ Res. 2007;101:839-47, with permission).

A

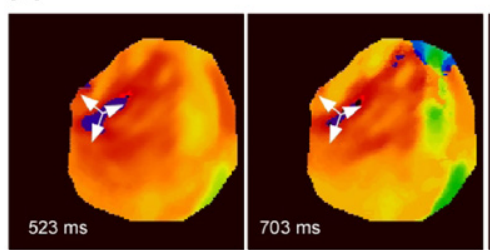

B

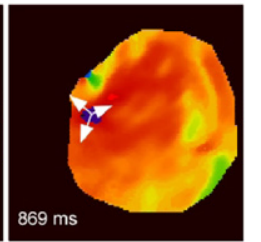

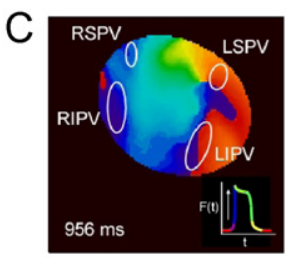
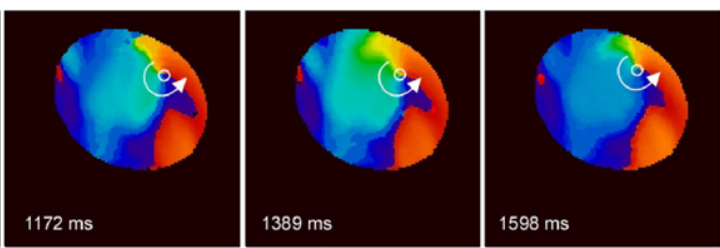

D

RAA

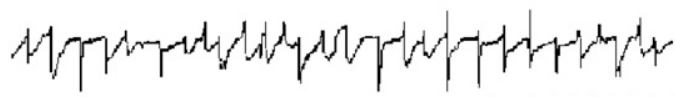

LSPV

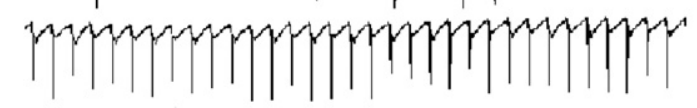

LAA

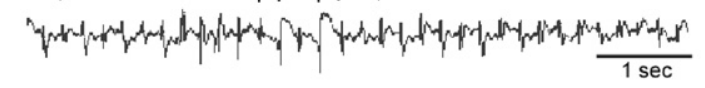

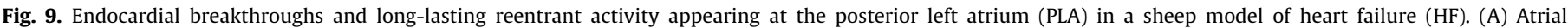

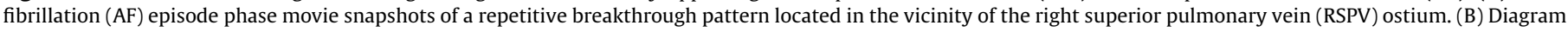

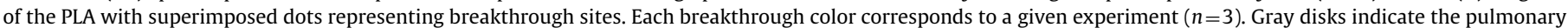

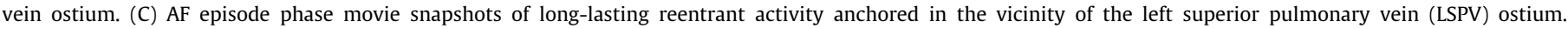
(D) Corresponding right atrial appendage (RAA), LSPV, and left atrial appendage (LAA) electrograms.

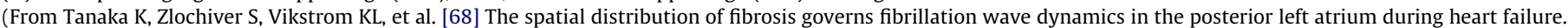
Circ Res. 2007;101:839-47, with permission). 
AF dynamics in this heart failure model are governed by the distribution of fibrosis.

\section{Conclusions}

The experimental results in isolated sheep hearts presented herein clearly demonstrate that self-sustained rotors exist in the atria. Such rotors are the high frequency sources that give rise to the complex patterns of activation that characterize AF. More specifically, our results suggest that the 3-D dynamics of the highfrequency rotors that generate scroll waves in the atrium determine the organization of $\mathrm{AF}$. It follows that understanding the dynamics of 3-D scroll waves is a crucial step that is needed for the development of novel anti-arrhythmic drugs and more effective ablative therapies. Importantly, recent work in patients gives credence to the idea that mother rotors are capable of maintaining AF in the human heart [25]. However, at this point it is too early to tell whether our observations of 3-D scroll waves in sheep hearts relate to the mechanisms of long-term persistent AF (12 months or longer) in humans. Nevertheless, we propose that the use of persistent AF models in animals together with the use of high-resolution mapping for the localization of rotors in the human heart will certainly help elucidate the mechanisms of AF and hopefully advance its treatment.

\section{Funding sources}

This work was supported by NHLBI grants (Grant numbers PO1 HL039707, PO1 HL087226, and RO1 HL070074 to J.J.) and Heart Rhythm Society Fellowship Award provided by The Fellowship of Japan Heart Foundation/The Japanese Society of Electrocardiology to M.Y.

\section{Conflict of interest}

All authors have no conflict of interest that should be disclosed.

\section{Acknowledgments}

We thank Dr. Haruo Honjo for fruitful discussion regarding this work.

\section{References}

[1] Chen LY, Shen WK. Epidemiology of atrial fibrillation: a current perspective. Heart Rhythm 2007;4:S1-6.

2] Inoue $\mathrm{H}$, Fujiki A, Origasa $\mathrm{H}$, et al. Prevalence of atrial fibrillation in the general population of Japan: an analysis based on periodic health examination. Int J Cardiol 2009;137:102-7.

[3] Ohsawa M, Okayama A, Sakata K, et al. Rapid increase in estimated number of persons with atrial fibrillation in Japan: an analysis from national surveys on cardiovascular diseases in 1980, 1990 and 2000. J Epidemiol 2005;15: 194-6.

[4] Nattel S, Maguy A, Le Bouter S, Yeh YH. Arrhythmogenic ion-channel remodeling in the heart: heart failure, myocardial infarction, and atrial fibrillation. Physiol Rev 2007;87:425-56.

[5] Wijffels MC, Kirchhof CJ, Dorland R, Allessie MA. Atrial fibrillation begets atrial fibrillation. A study in awake chronically instrumented goats. Circulation 1995;92:1954-68.

[6] Wijffels MC, Kirchhof CJ, Dorland R, Power J, Allessie MA. Electrical remodeling due to atrial fibrillation in chronically instrumented conscious goats: roles of neurohumoral changes, ischemia, atrial stretch, and high rate of electrical activation. Circulation 1997;96:3710-20.

[7] Haissaguerre M, Jais P, Shah DC, et al. Spontaneous initiation of atrial fibrillation by ectopic beats originating in the pulmonary veins. N Engl J Med 1998;339:659-66
[8] Jais P, Haissaguerre M, Shah DC, et al. A focal source of atrial fibrillation treated by discrete radiofrequency ablation. Circulation 1997;95:572-6.

[9] Manyari DE, Patterson C, Johnson D, Melendez L, Kostuk WJ, Cape RD. Atrial and ventricular arrhythmias in asymptomatic active elderly subjects: correlation with left atrial size and left ventricular mass. Am Heart 1990;119:1069-76.

[10] Benjamin EJ, Levy D, Vaziri SM, D’Agostino RB, Belanger AJ, Wolf PA Independent risk factors for atrial fibrillation in a population-based cohort The Framingham heart study. J Am Med Assoc 1994;271:840-4.

[11] Lewis T. The mechanism and graphic registration of the heart beat. 3rd ed. London: Shaw; 1925.

[12] Moe G. On the multiple wavelet hypothesis of atrial fibrillation. Arch Int Pharmacodyn Ther 1962:140:183-8.

[13] Moe GK, Abildskov JA. Atrial fibrillation as a self-sustaining arrhythmia independent of focal discharge. Am Heart J 1959;58:59-70.

14] Allessie M, Lammers W, Bonke F, Hollen J. Experimental evaluation of Moe's multiple wavelet hypothesis of atrial fibrillation. In: Zaipes D, Jalife J, editors Cardiac Electrophysiology and Arrhythmia. Orlando, FL: Grune and Stratton 1985. p. 265-75.

[15] Cox JL. The surgical treatment of atrial fibrillation. IV. Surgical technique. J Thorac Cardiovasc Surg 1991;101:584-92.

[16] Nademanee K, McKenzie J, Kosar E, et al. A new approach for catheter ablation of atrial fibrillation: mapping of the electrophysiologic substrate. J Am Coll Cardiol 2004:43:2044-53.

[17] Winfree AT. Spiral waves of chemical activity. Science 1972;175:634-6.

[18] Jalife J. Ventricular fibrillation: mechanisms of initiation and maintenance. Annu Rev Physiol 2000:62:25-50.

[19] Davidenko JM, Pertsov AV, Salomonsz R, Baxter W, Jalife J. Stationary and drifting spiral waves of excitation in isolated cardiac muscle. Nature 1992;355:349-51.

[20] Jalife J. Deja vu in the theories of atrial fibrillation dynamics. Cardiovasc Res 2011;89:766-75.

[21] Jalife J, Berenfeld O, Mansour M. Mother rotors and fibrillatory conduction: a mechanism of atrial fibrillation. Cardiovasc Res 2002:54:204-16.

[22] Atienza F, Almendral J, Moreno J, et al. Activation of inward rectifier potassium channels accelerates atrial fibrillation in humans: evidence for a reentrant mechanism. Circulation 2006;114:2434-42.

[23] Atienza F, Calvo D, Almendral J, et al. Mechanisms of fractionated electrograms formation in the posterior left atrium during paroxysmal atrial fibrillation in humans. J Am Coll Cardiol 2011;57:1081-92.

24] Sanders P, Berenfeld O, Hocini M, et al. Spectral analysis identifies sites of high-frequency activity maintaining atrial fibrillation in humans. Circulation 2005;112:789-97.

25] Narayan S, Patel J, Mulpuru S, Krummen D. Focal impulse and rotor modulation (FIRM) ablation of sustaining rotors abruptly terminates persistent atrial fibrillation to sinus rhythm with elimination on followup. Heart Rhythm, in press.

[26] Yamazaki M, Mironov S, Taravant C, et al. Heterogeneous atrial wall thickness and stretch promote scroll waves anchoring during atrial fibrillation. Cardiovasc Res 2012;94:48-57.

[27] Gray RA, Jalife J, Panfilov AV, et al. Mechanisms of cardiac fibrillation. Science 1995:270:1222-3 [Author reply 1224-1225].

[28] Ikeda T, Uchida T, Hough D, et al. Mechanism of spontaneous termination of functional reentry in isolated canine right atrium. Evidence for the presence of an excitable but nonexcited core. Circulation 1996;94:1962-73.

[29] Mandapati R, Skanes A, Chen J, Berenfeld O, Jalife J. Stable microreentrant sources as a mechanism of atrial fibrillation in the isolated sheep heart Circulation 2000;101:194-9.

[30] Mansour M, Mandapati R, Berenfeld O, Chen J, Samie FH, Jalife J. Left-to-right gradient of atrial frequencies during acute atrial fibrillation in the isolated sheep heart. Circulation 2001;103:2631-6.

[31] Filgueiras-Rama D, Martins RP, Ennis SR, et al. High-resolution endocardia and epicardial optical mapping in a sheep model of stretch-induced atrial fibrillation. J Vis Exp 2011;53:3103.

[32] Kalifa J, Klos M, Zlochiver S, et al. Endoscopic fluorescence mapping of the left atrium: a novel experimental approach for high resolution endocardial mapping in the intact heart. Heart Rhythm 2007;4:916-24.

[33] Gray RA, Pertsov AM, Jalife J. Spatial and temporal organization during cardiac fibrillation. Nature 1998;392:75-8.

[34] Warren M, Guha PK, Berenfeld O, et al. Blockade of the inward rectifying potassium current terminates ventricular fibrillation in the guinea pig heart. J Cardiovasc Electrophysiol 2003;14:621-31.

[35] Yamazaki M, Morgenstern S, Klos M, Campbell K, Buerkel D, Kalifa J. Left atrial coronary perfusion territories in isolated sheep hearts: implications for atrial fibrillation maintenance. Heart Rhythm 2010;7:1501-8.

[36] Walton RD, Mitrea BG, Pertsov AM, Bernus O. A novel near-infrared voltagesensitive dye reveals the action potential wavefront orientation at increased depths of cardiac tissue. Conf Proc IEEE Eng Med Soc 2009;2009:4523-6.

[37] Berenfeld O, Wellner M, Jalife J, Pertsov AM. Shaping of a scroll wave filament by cardiac fibers. Phys Rev E Stat Nonlinear Soft Matter Phys 2001;63: 061901.

[38] Wellner M, Berenfeld O, Jalife J, Pertsov AM. Minimal principle for rotor filaments. Proc Natl Acad Sci USA 2002;99:8015-8.

[39] Pertsov AM. Three-dimensional vortex-like reentry. In: Zipes DP, Jalife J, editors. From Cell to Bedside. 3 ed.. Philadelphia, Pa: WB Saunders; 2000. p. $403-10$. 
[40] Jalife J, Delmar M, Anumonwo JM, Berenfeld O, Rotors Kalifa J. Spirals, and scroll waves in the heart basic cardiac electrophysiology for the clinician. 2 ed. Oxford, UK: Wiley-blackwell; 2009.

[41] Cristal N, Peterburg I, Szwarcberg J. Atrial fibrillation developing in the acute phase of myocardial infarction. Prognostic implications. Chest 1976;70:8-11.

[42] Vaziri SM, Larson MG, Benjamin EJ, Levy D. Echocardiographic predictors of nonrheumatic atrial fibrillation. The Framingham heart study. Circulation 1994;89:724-30.

[43] Takase B, Nagata M, Matsui T, et al. Pulmonary vein dimensions and variation of branching pattern in patients with paroxysmal atrial fibrillation using magnetic resonance angiography. Jpn Heart J 2004;45:81-92.

[44] Yamane T, Shah DC, Jais P, et al. Dilatation as a marker of pulmonary veins initiating atrial fibrillation. J Interv Card Electrophysiol 2002;6:245-9.

[45] Kalifa J, Jalife J, Zaitsev AV, et al. Intra-atrial pressure increases rate and organization of waves emanating from the superior pulmonary veins during atrial fibrillation. Circulation 2003;108:668-71.

[46] Yamazaki M, Vaquero LM, Hou L, et al. Mechanisms of stretch-induced atrial fibrillation in the presence and the absence of adrenocholinergic stimulation: interplay between rotors and focal discharges. Heart Rhythm 2009;6: 1009-1017.

[47] Ravelli F, Allessie M. Effects of atrial dilatation on refractory period and vulnerability to atrial fibrillation in the isolated Langendorff-perfused rabbit heart. Circulation 1997;96:1686-95.

[48] Burashnikov A, Antzelevitch C. Reinduction of atrial fibrillation immediately after termination of the arrhythmia is mediated by late phase 3 early afterdepolarization-induced triggered activity. Circulation 2003;107: 2355-2360.

[49] Coumel P. Cardiac arrhythmias and the autonomic nervous system. J Cardiovasc Electrophysiol 1993;4:338-55.

[50] Patterson E, Po SS, Scherlag BJ, Lazzara R. Triggered firing in pulmonary veins initiated by in vitro autonomic nerve stimulation. Heart Rhythm 2005;2:624-31.

[51] Schauerte P, Scherlag BJ, Pitha J, et al. Catheter ablation of cardiac autonomic nerves for prevention of vagal atrial fibrillation. Circulation 2000;102: 2774-2780.

[52] Burashnikov A, Antzelevitch C. Late-phase 3 EAD. A unique mechanism contributing to initiation of atrial fibrillation. Pacing Clin Electrophysio 2006;29:290-5.

[53] Berenfeld O, Zaitsev AV, Mironov SF, Pertsov AM, Jalife J. Frequencydependent breakdown of wave propagation into fibrillatory conduction across the pectinate muscle network in the isolated sheep right atrium. Circ Res 2002;90:1173-80.

[54] Gray RA, Pertsov AM, Jalife J. Incomplete reentry and epicardial breakthrough patterns during atrial fibrillation in the sheep heart. Circulation 1996;94: 2649-2661.
[55] Wu TJ, Yashima M, Xie F, et al. Role of pectinate muscle bundles in the generation and maintenance of intra-atrial reentry: potential implications for the mechanism of conversion between atrial fibrillation and atrial flutter. Circ Res 1998;83:448-62.

[56] Gaspo R, Sun H, Fareh S, et al. Dihydropyridine and beta adrenergic receptor binding in dogs with tachycardia-induced atrial fibrillation. Cardiovasc Res 1999;42:434-42.

[57] Yue L, Feng J, Gaspo R, Li GR, Wang Z, Nattel S. Ionic remodeling underlying action potential changes in a canine model of atrial fibrillation. Circ Res 1997;81:512-25.

[58] Derakhchan K, Li D, Courtemanche M, et al. Method for simultaneous epicardial and endocardial mapping of in vivo canine heart: application to atrial conduction properties and arrhythmia mechanisms. J Cardiovasc Electrophysiol 2001;12:548-55.

[59] Eckstein J, Maesen B, Linz D, et al. Time course and mechanisms of endoepicardial electrical dissociation during atrial fibrillation in the goat. Cardiovasc Res 2011;89:816-24.

[60] Courtemanche M, Ramirez RJ, Nattel S. Ionic targets for drug therapy and atrial fibrillation-induced electrical remodeling: insights from a mathematical model. Cardiovasc Res 1999;42:477-89.

[61] Pandit SV, Berenfeld O, Anumonwo JM, et al. Ionic determinants of functional reentry in a 2-D model of human atrial cells during simulated chronic atrial fibrillation. Biophys J 2005;88:3806-21.

[62] Kamkin A, Kiseleva I, Wagner KD, et al. Characterization of stretch-activated ion currents in isolated atrial myocytes from human hearts. Pflugers Arch 2003;446:339-46.

[63] Mathew J, Hunsberger S, Fleg J, Mc Sherry F, Williford W, Yusuf S. Incidence, predictive factors, and prognostic significance of supraventricular tachyarrhythmias in congestive heart failure. Chest 2000;118:914-22.

[64] Li D, Fareh S, Leung TK, Nattel S. Promotion of atrial fibrillation by heart failure in dogs: atrial remodeling of a different sort. Circulation 1999;100: $87-95$.

[65] Verheule S, Sato $\mathrm{T}$, Everett $\mathrm{Tt}$, et al. Increased vulnerability to atrial fibrillation in transgenic mice with selective atrial fibrosis caused by overexpression of TGF-beta1. Circ Res 2004;94:1458-65.

[66] Fenelon G, Shepard RK, Stambler BS. Focal origin of atrial tachycardia in dogs with rapid ventricular pacing-induced heart failure. J Cardiovasc Electrophysiol 2003;14:1093-102.

[67] Stambler BS, Fenelon G, Shepard RK, Clemo HF, Guiraudon CM. Characterization of sustained atrial tachycardia in dogs with rapid ventricular pacinginduced heart failure. J Cardiovasc Electrophysiol 2003;14:499-507.

[68] Tanaka K, Zlochiver S, Vikstrom KL, et al. Spatial distribution of fibrosis governs fibrillation wave dynamics in the posterior left atrium during heart failure. Circ Res 2007;101:839-47. 